\title{
Analysis of agricultural meteorological disasters in Shaanxi Province from 1981 to 2018 and their impact on grain production
}

\author{
Wang Chunlin ${ }^{1,2,3,4^{*}}$, Xiong Yufei ${ }^{1,2,3,4}$ \\ ${ }^{1}$ Shaanxi Provincial Land Engineering Construction Group Co., Ltd., Xi'an 710075, China \\ ${ }^{2}$ Institute of Land Engineering and Technology, Shaanxi Provincial Land Engineering Construction \\ Group Co., Ltd., Xi'an 710075, China \\ ${ }^{3}$ Key Laboratory of Degraded and Unused Land Consolidation Engineering, the Ministry of Natural \\ Resources, Xi'an 710075, China \\ ${ }^{4}$ Shaanxi Provincial Land Consolidation Engineering Technology Research Center, Xi'an 710075, \\ China
}

\begin{abstract}
This paper studied the change characteristics of four types of agricultural meteorological disasters such as flood, drought, hail and freeze and their impact on the total grain yield of Shaanxi Province. Based on the agricultural statistical data of Shaanxi Province from 1981 to 2018, the disaster-affected rate, disaster-suffered rate and disaster intensity index, as well as the grey correlation analysis method were used to analyze the agricultural meteorological disasters and their impact on grain yield in Shaanxi Province from 1981 to 2018. The results show that drought has the largest disaster-affected area and disaster-suffered area in Shaanxi Province, but the disaster-affected rate and disaster-suffered rate increases first and then decreases. The difference of disaster intensity among flood, drought and hail is relatively small, among which the drought disaster has the strongest disaster intensity and the freeze has a relatively weak disaster intensity. The grey correlation analysis show that the affected area of hail disasters has the greatest impact on the total grain yield of Shaanxi Province, followed by drought and flood, and the affected area of freeze disaster has a relatively small impact on the total grain yield.
\end{abstract}

Keywords: Shaanxi Province, agrometeorological disaster, grain yield

\section{Introdution}

Agricultural meteorological disasters have a high frequency and a wide range of effects, which is an important factor restricting grain production. ${ }^{[1]}$ The loss caused by agrometeorological disasters in China is huge every year. ${ }^{[2]}$ Shaanxi Province is a major agricultural province in Northwest China, in which Guanzhong Plain is an important grain producing area. Due to the transition zone from the humid region in the east to the arid region in the west, the climate difference in Shaanxi Province is large and variable, with the

* Corresponding author e-mail: chunlinyx86@163.com 
grain production being greatly affected by meteorological disasters. ${ }^{[3]}$ In addition, the construction of rural meteorological service system in Shaanxi province lags behind, the coverage of early warning information for meteorological disasters is not high. Every year, meteorological disasters such as drought, hail, low temperature, and heavy rain cause significant losses to agricultural production. The annual average disaster area of crops caused by flood and drought disasters accounts for more than $80 \%$ of the annual average disaster area caused by natural disasters. ${ }^{[4]} \mathrm{Li}$ Jianping et $\mathrm{al}^{[5]}$ predicted the trend of grain production and grain security in Shaanxi Province, pointing out that climate fluctuations are the main reason for fluctuations of grain per unit area yield, the key to the increase of grain production in Shaanxi Province is to alleviate the impact of meteorological disasters on agricultural production. Under the background of climate warming, the uncertainty of extreme weather events in Shaanxi Province has increased, ${ }^{[6-8]}$ which leads to the further increase of the instability of grain production in Shaanxi Province. Therefore, to ensure the stable increase of grain production, making clear how the agricultural meteorological disasters change and knowing the impact of different agricultural meteorological disasters on grain production is indispensable.

At present, there are relatively few studies on the agrometeorological disasters and grain production in Shaanxi Province. The limited researches are focused on single-hazard species like the trend analysis and spatial distribution characteristics of drought and flood disasters. The researches on the intensity of the agrometeorological disasters in Shaanxi Province and the relationship between the intensity of the disasters and grain production are insufficient, especially the comparative studies on the different agrometeorological disasters in Shaanxi Province after 2010 are rarely reported. Based on the agricultural statistical data of Shaanxi Province from 1981 to 2018, this paper analyzes the change characteristics of flood, drought, hail and freeze disasters in Shaanxi Province in recent 38 years by the indicators of disaster rate and disaster intensity. According to the method of grey correlation analysis, the relationship between the affected area of various disasters and total grain yield is studied, aiming at gaining a clear idea of which is most harmful to the total grain yield of Shaanxi Province among the agrometeorological disasters, so as to provide scientific decision-making basis for grain security and disaster prevention and mitigation in Shaanxi Province.

\section{Materials and methods}

\subsection{Materials Source}

This paper selects the crop planting area, grain yield, the affected area of flood, drought, hail and freeze disasters of Shaanxi Province from 1981 to 2018. The statistical data comes from the National Bureau of Statistics of China (http://data.stats.gov.cn/). Howerer, the data on freeze disasters in 1981, 1984, 1986, and 1996 is missing, so that the analysis of this 4a is not got to be analyzed.

\subsection{Research methods}

\subsubsection{Disaster-affected rate and disaster-suffered rate}

When the agricultural department makes statistics of the disaster situation, it generally calculates the grain loss according to disaster-affected area and disaster-suffered area. Disaster-affected area refers to the sown area of grain whose yield is reduced by more than $10 \%$ of the normal yield under the influence of disasters. Disaster-suffered area refers to the 
sown area whose yield is reduced by more than $30 \%$ due to disasters. Since the grain planting area varies from year to year, for eliminating the impact of the interannual change of grain planting area on the change of disaster trends, it is necessary to calculate the disaster-affected rate and disaster-suffered rate of various disasters. ${ }^{[9]}$ The disaster-affected rate and the disaster-suffered rate can represent the size of the disaster area of the grain affected by meteorological disasters. The calculation formula is as follows.

$$
\begin{aligned}
& A_{i}=H_{1 i} / S_{i} \times 100 \% \\
& B_{i}=H_{2 i} / S_{i} \times 100 \%
\end{aligned}
$$

Where $A_{i}$ and $B_{i}$ is the disaster-affected rate and disaster-suffered rate of year $i . i=1981$, $1982, \ldots, 2018, H_{1 i}$ and $H_{2 i}$ is the disaster-affected area and disaster-suffered area of year $i$, $S_{i}$ is the grain sown area of year $i$.

\subsubsection{Disaster intensity index}

The disaster intensity index represents the disaster intensity of natural disasters on the production of grain per unit area, which is the proportion of the disaster-affected area to the disaster-suffered area in a certain period. ${ }^{[10]}$ The calculation formula is as follows.

$$
Z_{i}=H_{1 i} / H_{2 i} \times 100 \%
$$

Where $Z_{i}$ is the disaster intensity index of year i, $H_{l i}$ and $H_{2 i}$ is the disaster-affected area and disaster-suffered area of year $i$.

\subsubsection{Grey correlation analysis}

Grey correlation analysis is a multi-factor statistical analysis method that measures the degree of correlation between factors. Its basic principle is based on the grey process of grey system theory, calculating the correlation between different variables and quantitatively comparing the similarity or dissimilarity of development trends between factors to determine the process of leading factors and restricting factors. Gray correlation analysis is more capable of grasping the main contradictions than correlation analysis, which can reflect its biological significance in screening meteorological factors, so it is widely used in various fields of natural science. ${ }^{[1]}$ As a result, this paper uses the grey correlation degree to characterize the impact of various meteorological disasters on grain yield.

\section{Results and analysis}

\subsection{Characteristics of agrometeorological disasters in Shaanxi Province}

\subsubsection{Disaster-affected rate and disaster-suffered rate}

The change of flood, drought, hail and freeze on disaster-affected rate and disaster-suffered rate in Shaanxi Province from 1981 to 2018 is shown in Figure 1. It can be seen from Fig.1(a) that the flood disaster-affected rate and the disaster-suffered rate fluctuate periodically as a whole. In recent 38 years, the annual average disaster-affected rate of 
flood disaster is $8.8 \%$, the annual average disaster-suffered rate is $4.6 \%$. In 2003 , the disaster-affected rate and the disaster-suffered rate of flood disaster are the largest and theirs values are $30.3 \%$ and $16.8 \%$ respectively. During 2001-2010, the disaster-affected rate and the disaster-suffered rate of flood disaster fluctuated the most, between $1.6 \%-30.3 \%$ and $0.5 \%-16.8 \%$ respectively. Both the disaster-affected rate and the disaster-suffered rate of flood disaster show a decreasing trend after 2010.
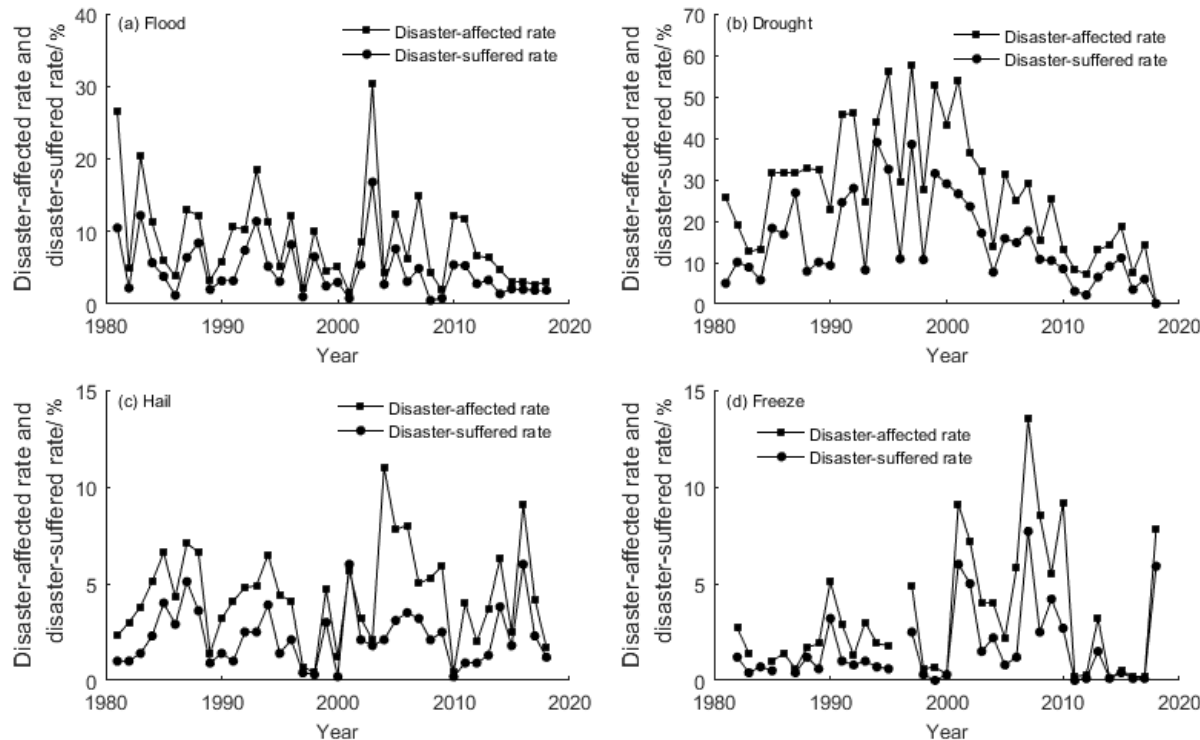

Fig.1 The changes of flood (a), drought (b), hail (c), freeze (d) disaster-affected rate and disaster-suffered rate in 1981-2018 in Shaanxi Province

It can be seen from Fig.1 (b) that the disaster-affected rate and the disaster-suffered rate of drought disaster show a trend of increasing first and then decrease. In the 1990s, the fluctuation of the disaster-affected rate and the disaster-suffered rate of drought disaster is relatively frequent and high. In recent 38 years, the annual average disaster-affected rate of drought is $27.3 \%$ and the annual average disaster-suffered rate is $14.9 \%$. In 1997 , the disaster-affected rate of drought was the largest, reaching 57.7\%. In 1994, the disaster-suffered rate of drought was the largest, reaching $39.0 \%$. The drought disaster-affected rate fluctuated the most from 2001 to 2010 , ranging from $13.2 \%$ to $54.0 \%$. In the $1990 \mathrm{~s}$, the drought-suffered rate fluctuated the most, ranging from $8.2 \%$ to $39.0 \%$. Since the $1990 \mathrm{~s}$, the disaster-affected rate and the disaster-suffered rate of drought disaster have been decreasing.

It can be seen from Fig.1(c) that the disaster-affected rate and the disaster-suffered rate of hail disaster have relatively stable changes in the whole study period. In recent 38 years, the annual average disaster-affected rate of hail disaster is $4.4 \%$ and the annual average disaster-suffered rate is $2.3 \%$. In 2004 , the disaster-affected rate of hail disaster is the largest, reaching $11.0 \%$. In 2001 and 2016, the disaster-suffered rate of hail disaster is the largest, reaching $6.0 \%$. During 2001-2010, the fluctuation range of the disaster-affected rate and the disaster-suffered rate of hail disaster is the largest, which are between $0.4 \%-11.0 \%$ and $0.2 \%-6.0 \%$ respectively. The disaster-affected rate and the disaster-suffered rate of hail disaster have been on the rise after 2010 .

Due to the lack of data on freeze disaster in 1981, 1984, 1986 and 1996, no analysis has been made on the disaster-affected rate and the disaster-suffered rate of freeze disaster in this 4 year. It can be seen from Fig.1(d) that the annual average disaster-affected rate of 
freeze disaster is $3.0 \%$ and the annual average disaster-suffered rate is $1.5 \%$ in recent 34 years. The disaster-affected rate and the disaster-suffered rate of freeze disaster in 2007 are the largest, reaching $13.5 \%$ and $7.7 \%$ respectively. During 2001-2010, the disaster-affected rate and the disaster-suffered rate of freeze disaster fluctuated the most, ranging from $2.2 \%$ $-13.5 \%$ and $0.8 \%-7.7 \%$ respectively. In addition, the annual average disaster-affected rate and the annual average disaster-suffered rate of the freeze disaster during 2001-2010 are 6.9\% and $3.4 \%$ respectively, much higher than the annual average disaster-affected rate and the annual average disaster-suffered rate in recent 34 years.

\subsubsection{Disaster intensity index}

The change trend of disaster intensity index of flood, drought, hail and freeze disasters in Shaanxi Province from 1981 to 2018 is shown in Fig.2. It can be seen from Fig.2(a) that the change of flood disaster intensity index in Shaanxi Province has stage characteristics. The annual average flood disaster intensity index in recent 38 years is $52.3 \%$, among which 1988, 1992, 2015 and 2017 are high-intensity years and the disaster intensity indexes are $69.1 \%, 71.2 \%, 69.1 \%$ and $68.6 \%$ respectively. During the period of $2005-2015$, the intensity of flood disaster fluctuated the most, indaicating that the annual variation of disaster intensity was severe, with the disaster intensity being relatively weak. In the 1990s, the annual average disaster intensity index was the highest, reaching $56.4 \%$, when the flood disaster intensity was the largest.
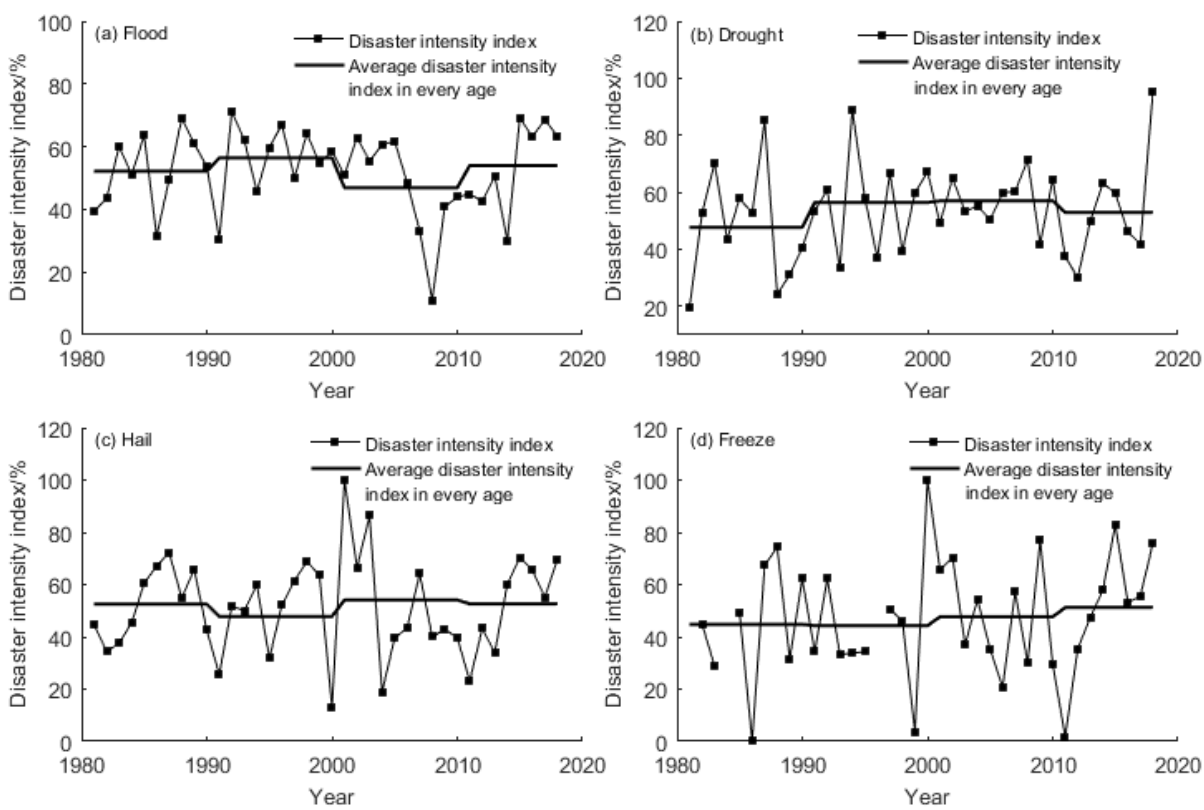

Fig.2 The change of disaster intensity index of flood (a), drought (b), hail (c) and freeze (d) in 1981-2018 in Shaanxi Province

It can be seen from Fig.2 (b) that the change of drought disaster intensity index in Shaanxi Province in the study period is relatively stable. The annual average drought disaster intensity index in recent 38 years is 53.6\%, among which 1987, 1994 and 2018 are high-intensity years and its disaster intensity indexes are $85.2 \%, 88.9 \%$ and $95.2 \%$ respectively. In the 1980 s, the intensity of drought disaster fluctuated the most, indaicating that the annual variation of disaster intensity changed drastically. During the 1990s and 
2001-2010, the intensity of drought disaster fluctuated less. The annual average disaster intensity index was $56.4 \%$ and $57.0 \%$ respectively, the disaster intensity is relatively large.

It can be seen from Fig.2(c) that the annual average disaster intensity index of hail disaster in recent 38 years is $51.7 \%$, among which 2001 and 2003 are high-intensity years and its disaster intensity indexes are $100 \%$ and $86.4 \%$ respectively. During $2000-2004$, the intensity of hail disaster changed dramatically. In the 1990s, the annual average disaster intensity index of hail disaster is the smallest and its value is $47.8 \%$. In other years, the annual average disaster intensity index of hail disaster had a little difference, indiacaing the change of disaster intensity was relatively stable.

It can be seen from Fig.2 (d) that the change of freeze disaster intensity index in Shaanxi Province is characterized by a gradual increase. In recent 38 years, the annual average disaster intensity index of freeze disaster is $47.0 \%$, among which 1988, 2000, 2002, 2009,2015 and 2018 are the high-intensity years and their disaster intensity index are more than $70 \%$. The disaster intensity index of freeze disaster fluctuates largely in the studied period, indaicating that the annual variation of disaster intensity changed drastically. During 2011-2018, the annual average disaster intensity index is the highest and its value is $51.2 \%$, when the freeze disaster is the largest.

\subsection{Correlation analysis of grain yield and agrometeorological disasters}

In the correlation analysis of the affected areas, the total grain yield of Shaanxi Province from 1981 to 2018 was used as a reference sequence, while the affected area of four types of agro-meteorological disasters was used as a comparative sequence to calculate the correlation between the total grain yield and the affected areas of four types of agrometeorological disasters. The results are shown in Table 1. According to the principle of grey relation analysis, the greater the correlation degree is, the greater the impact weight is, so that the greater the impact of this meteorological disaster factor on grain yield. It can be seen from table 1 that from the perspective of affected areas, the correlation between drought and the total grain yield of Shaanxi Province is the largest in 1980s, 1990s and 2001-2010, which are $0.814,0.785$ and 0.765 respectively, indicating that the impact of drought disaster affected area on the total grain yield of Shaanxi Province is the largest in this period.

Table 1 The correlation between the total grain yield and the affected areas of four types of agro-meteorological disasters

\begin{tabular}{|c|c|c|c|c|c|}
\hline Stage & Indacators & Flood & Drought & Hail & Freeze \\
\hline \multirow{2}{*}{$1981-1990$} & Correlation & 0.634 & 0.814 & 0.729 & 0.704 \\
\cline { 2 - 6 } & Sort & 4 & 1 & 2 & 3 \\
\hline \multirow{2}{*}{$1991-2000$} & Correlation & 0.683 & 0.785 & 0.618 & 0.634 \\
\cline { 2 - 6 } & Sort & 2 & 1 & 4 & 3 \\
\hline \multirow{2}{*}{$2001-2010$} & Correlation & 0.688 & 0.765 & 0.753 & 0.757 \\
\cline { 2 - 6 } & Sort & 4 & 1 & 3 & 2 \\
\hline \multirow{2}{*}{$2011-2018$} & Correlation & 0.828 & 0.827 & 0.845 & 0.652 \\
\cline { 2 - 6 } & Sort & 2 & 3 & 1 & 4 \\
\hline \multirow{2}{*}{$1981-2018$} & Correlation & 0.726 & 0.736 & 0.768 & 0.689 \\
\cline { 2 - 6 } & Sort & 3 & 2 & 1 & 4 \\
\hline
\end{tabular}

From 2011 to 2018, the order of the correlation degree between the disaster affected area of each agrometeorological disaster and the total grain yield is hail, flood, drought and freeze from high to low, which indicates that the impact of the disaster affected area of hail 
on the total grain yield of Shaanxi Province is the largest in the past eight years. During 1981-2018, the order of the correlation degree between the disaster affected area of each agrometeorological disaster and the total grain yield is hail, drought, flood and freeze from high to low, the correlation degree is $0.768,0.736,0.726$ and 0.689 respectively. It shows that the disaster affected area of hail disaster has the greatest impact on the total grain yield of Shaanxi Province in the past 38 years, followed by drought and flood. The affected area of freeze disaster has a relatively small impact on the total grain yield.

\section{Conclusion}

Based on the analysis of the characteristics of agrometeorological disasters in Shaanxi Province in the past 38 years, it can be seen that the change trend of disaster-affected rate and dissaster-suffered rate of flood, drought, hail and freeze disaster is relatively consistent, in which the disaster-affected rate and the disaster-suffered rate of drought are increasing first and then decreasing, while the disaster-affected rate and the disaster-suffered rate of freeze are increasing as a whole. Drought is the main agrometeorological disaster in Shaanxi Province. In the past 38 years, the disaster-affected rate of drought is much higher than other agrometeorological disasters in 33 years, with the disaster-suffered rate of drought being much higher than other agrometeorological disasters in 30 years, which indicates that the drought is the most widely affected agrometeorological disaster in Shaanxi Province. Compared with the change of disaster intensity index in different years, the annual average disaster intensity index of flood, drought, hail and freeze disaster in recent 38 years is $52.3 \%, 53.6 \%, 51.7 \%$ and $47.0 \%$ respectively, which shows that the difference in the disaster intensity is relatively small, with the drought having the strongest disaster intensity and the freeze having a relatively weak disaster intensity.

Different agrometeorological disasters have different impacts on grain yield. From the perspective of disaster affected area, the impact of the disaster affected area of hail on the total grain yield of Shaanxi Province is the largest in the past 38 years, followed by drought and flood, the affected area of freeze disaster has a relatively small impact on the total grain yield.

\section{References}

1. Luan Jian, Zhou Yuxi. An estimation of grain loss caused by natural disasters in Shandong Province based on grey prediction model and its correlation with disasters[J]. Research of Agricultural Modernization, 2016, 37(06): 1068-1075.

2. WU Jidong, $\mathrm{Fu} \mathrm{Yu,} \mathrm{Zhang} \mathrm{Jie,} \mathrm{et} \mathrm{al.} \mathrm{Meteorological} \mathrm{Disaster} \mathrm{Trend} \mathrm{Analysis} \mathrm{in} \mathrm{China:}$ 1949-2013[J]. Journal of Natural Resources, 2014, 29(09): 1520-1530.

3. Gao Maosheng, Fan Jianzhong, Wu Qingli. Influence of drought and flood disasters on grain production in Shaanxi Province[J]. Journal of China Agricultural University, 2012,17(03): 149-153.

4. Gao Maosheng, Fan Jianzhong, Wu Qingli. Analysis on the Characteristics of Natural Disasters and the Disaster Loss in Shaanxi Province[J]. Acta Agriculturae Boreali-occidentalis Sinica, 2012, 21(07): 202-206.

5. LI Jianping, Shangguan Zhouping. Trend forecast of grain production and food security in Shaanxi Province[J]. Agricultural Research in the Arid Areas, 2011, 29(04): 245-251.

6. Wang Wenyan, Wang Li, Li Guoping, et al. Temporal and Spatial Characteristics of Extreme Precipitation in Flood Season in Shaanxi Province During 1961-2013[J]. 
Journal of Arid Meteorology, 2016, 34(06): 952-957.

7. Wang Na, Wang Qi, Fang Feng, et al. Characteristics of spatial and temporal variation of extreme no rain days in each season in Shaanxi province[J]. Agricultural Research in the Arid Areas, 2014, 32(06): 221-228.

8. Jiang Chuangye, Cai Xinling, Wu Suliang, et al. Spatio temporal Evolution of Extreme Precipitation Events in Shaanxi Province during the Period 1961 -2007[J]. Arid Zone Research, 2011, 28(01): 151-157.

9. Wen Danping, Li Jing, He Pengfei. Grey correlation analysis of agro-meteorological disasters andsoybean yield in Heilongjiang province[J]. Journal of Natural Disasters, 2017, 26(04): 56-62.

10. Li Zhiguo. Influence of agricultural meteorological disasters on grain production in Henanprovince[J]. Journal of Arid Land Resources and Environment, 2013, 27(05): 126-130.

11. Yang Chunyan, Sheng Weishou, Li Haidong. Response of grain yield in Tibet to climate and cultivated land change during 1985-2010[J]. Transactions of the Chinese Society of Agricultural Engineering, 2015, 31(17): 261-269. 